\title{
Estudio diagnóstico del bienestar de bovinos durante el transporte a una planta de beneficio de la región caribe de Colombia
}

\section{Diagnostic study of bovine welfare during transport to a beneficiary plant in the Caribbean region of Colombia}

\author{
Pérez -Viloria Juan ${ }^{1 *}$ Zoo, Roqueme-Blanco Wilberto ${ }^{1}$ Zoo, \\ Patiño -Pardo René ${ }^{1}$ Dr.Sc, Botero- Arango Luz ${ }^{1}$ M.Sc. \\ ${ }^{1}$ Universidad de Sucre. Facultad De Ciencias Agropecuarias, Programa de Zootecnia, \\ Semillero de Investigación INNOVAR, Grupo de Investigación en Biodiversidad Tropical,Sincelejo, Colombia
}

Keywords:

Animal welfare; animal management; Caribbean Region; cattle

\begin{abstract}
A diagnostic animal welfare (AW) study was carried out during the terrestrial transport of cattle to a slaughtering plant in the Caribbean region of Colombia. A cross sectional analytical study was carried out on 20 drivers, evaluating demographic variables, attitude towards cattle and the management. Data of 59 trips (588 cattle) were analyzed, evaluating the conditions of the vehicles and determining load density, weight heterogeneity, distance traveled, waiting time before landing and the employee at the landing. Parts of vehicles are not used exclusively for transporting cattle and do not fully comply with the provisions of the ICA. Load densities were lower than those recommended, with values of $320.5 \mathrm{~kg} / \mathrm{m}^{2}(\mathrm{SD}=6.3)$ and $285.2 \mathrm{~kg} / \mathrm{m}^{2}(\mathrm{SD}=83.48)$ for small and large vehicles, respectively. Distance traveled was $21.6 \mathrm{~km}$, on average. The average total wait and discharge times were 11.1 and $2.8 \mathrm{~min}$, respectively. The distance traveled, the charge density and the discharge time were correlated with the number of hematomas and the $\mathrm{pH}$ of the cold carcass. Failures that have a negative effect on the welfare of cattle during transportation and unloading in the dressing plant and on the quality of the carcass were evidenced, together with the lack of training of the drivers in the AW.
\end{abstract}

\section{Resumen}

Se realizó un estudio diagnóstico de bienestar animal (BA) durante el transporte terrestre de vacunos hacia una planta de faenado de la región Caribe de Colombia. Se hizo un estudio transversal analítico a 20 transportistas, evaluando variables demográficas, de actitud frente a los bovinos y del manejo dado. Se analizaron datos de 59 viajes (588 vacunos), evaluando las condiciones de los vehículos y determinando densidad de carga, heterogeneidad en peso, distancia recorrida, tiempo de espera antes del desembarque y el empleado en el desembarque. Parte de vehículos no se usan exclusivamente para el transporte de vacunos y no cumplen totalmente con lo dispuesto por el ICA. Las densidades de carga fueron inferiores a las recomendadas, con valores de $320,5 \mathrm{~kg} / \mathrm{m}^{2}(\mathrm{DE}=6,3)$ y $285,2 \mathrm{~kg} / \mathrm{m}^{2}$ (DE=83,48), para vehículos pequeños y grandes, respectivamente y la distancia recorrida fue de $21,6 \mathrm{~km}$, en promedio. Los tiempos promedios de espera total y de descargue fueron de $11,1 \mathrm{y}$ 2.8 min., respectivamente. La distancia recorrida, la densidad de carga y el tiempo de descargue se correlacionaron con el número de hematomas y el $\mathrm{pH}$ de la canal fría. Se evidenciaron fallas que repercuten negativamente en el bienestar de vacunos durante el transporte y descargue en la planta de faenado y sobre la calidad de la canal, aunado a la falta de capacitación de los conductores en el tema del BA. 


\section{Introducción}

La organización mundial de sanidad animal - OIE (2010) plantea que el BA es la forma como los animales se desarrollan y afrontan las distintas condiciones de su hábitat, y que están en bienestar cuando se encuentran en buen estado de salud, bien nutridos y en condiciones tales que ellos puedan expresar su comportamiento natural. Sin embargo, la definición más utilizada a nivel mundial es la propuesta por BROOM $(1986 ; 1991)$ quien define el bienestar como el estado de un individuo respecto a sus intentos de afrontar las condiciones que los rodean.

En Colombia, el concepto de bienestar animal (BA) se ha ido incluyendo atreves de la resolución ICA 002341 de 2007, por la cual se reglamentan las condiciones sanitarias y de inocuidad en la producción primaria de ganado bovino y bufalino destinado al sacrificio para consumo humano. De igual manera, este concepto se incluyó en la movilización o transporte bovino mediante el decreto 414 de 2007. El Ministerio de la Protección Social también incluyó ese concepto de bienestar en el proceso de faenado para la creación de la normatividad respectiva (Decreto 1500 de 2007) y la Resolución 2905 de 2007 (ROMERO y SANCHEZ, 2012).

DELUQUE y DUSSAN (2009) plantean que el transporte animal es la actividad en la cual los animales van a ser movilizados por diversas causas. En los países de América del Sur el transporte de ganado vacuno se realiza principalmente por vía terrestre (GALLO, 2008a). El transporte terrestre en camiones, al igual que el manejo que se le da a los animales en finca como movilización o arreo, son los eventos más comunes a los cuales se someten los animales destinados para el consumo humano (GALLO, 2008b). En este sentido se ha observado que en varios países Latino Americanos se prefiere reducir la disponibilidad de espacio por animal dentro del vehículo, ya sea por presiones comerciales o para disminuir costos de transporte (GALLO et al., 2005). Cuando la densidad de carga está por encima de los límites exigidos, se reduce el espacio libre disponible para cada animal y la libertad de movimiento, impidiendo que los animales puedan mantener su balance (GALLO et al., 2005). En Colombia, ROMERO et al. (2012) encontraron en diferentes regiones del país densidades de carga entre los 300 y $350 \mathrm{~kg} / \mathrm{m}^{2}$, las cuales resultaron ser bajas, ya que encontraron hematomas y contusiones en los animales, debido al espacio excedente en el vehículo. En este sentido, densidades altas como bajas afectan el BA, debido a la generación de contusiones (GALLO, 2008b). La OIE (2015) indica que los conductores deben de estar capacitados con respecto al BA de la especie que transportan, y el ICA, por medio de la resolución 002341 de 2007, plantea que el conductor o transportador de animales para abasto deberá demostrar que ha sido capacitado y que es competente para la movilizar animales en pie.

En cuanto al descargue de los animales en la planta de faenado se recomienda que el tiempo de espera sea de hasta 15 minutos, y que en lo posible que nunca sobrepase 20 minutos (GRANDIN, 2003; 2010). Algunos autores afirman que el embarque en finca y el desembarque en las plantas de faenado pueden ser eventos más estresantes que el mismo viaje (MARÍA et al., 2004). En el desembarque de los animales en los frigoríficos es común encontrar rampas en malas condiciones, sin sistemas antideslizantes, que hacen que los animales resbalen y caigan, generando nerviosismo en ellos y a veces daños físicos graves (HUERTAS, 2009).

En el caso de la Región Caribe de Colombia, por ser una zona en donde la ganadería vacuna es uno de los principales renglones económicos del sector agrario, se hace necesario llevar a cabo estudios sobre BA, por aspectos éticos y para atender la exigencia de mercados internacionales comprometidos con el BA.

El objetivo del estudio fue realizar un diagnóstico del BA a partir de la caracterización y evaluación del manejo de vacunos durante el transporte y descargue en una planta de faenado de la región Caribe de Colombia.

\section{Material y métodos}

Local de estudio: La investigación se realizó en una planta de beneficio de bovinos de la región Caribe de Colombia y autorizada por el INVIMA. Época de estudio: Las observaciones se realizaron entre los meses de junio a julio del año 2015. 
Estudio etnográfico: Se realizó un estudio etnográfico transversal analítico, mediante la aplicación de un formulario-encuesta, previamente diseñado, a 20 transportadores dispuestos a contribuir en la actividad, quienes fueron informados previamente sobre el objetivo del trabajo. Se evaluaron variables demográficas (edad de los conductores, nivel de escolaridad, experiencia en el oficio, entre otras.), y se indagó, también, sobre el tipo de manejo realizado a los animales durante el transporte. Paralelo al trabajo de toma de muestras y medición de variables, se llevó a cabo un trabajo de campo en donde se aplicaron variables técnicas de recolección de información, para tratar de explicar el manejo de los diferentes actores de la ganadería impartían a los animales y poder explicar cómo incidió sobre el bienestar o no del animal durante la etapa de transporte; estas técnicas fueron: Observación dirigida, que según ALAMINOS (2005), permite focalizar la atención de manera intencional sobre algunos segmentos de la realidad que se estudia; documentación fotográfica y videos elaborados durante el ejercicio de observación, para para permitir una interpretación analítica y sintética de algunos hechos u observaciones que desbordan las declaraciones de los entrevistados (LOZANO, 2008); las anotaciones de campo, y la entrevista semi-estructurada. Como se trataba de personas con diferentes niveles culturales y de educación, se siguió el sistema de entablar conversación sobre el BA, para luego analizar todas las entrevistas y extraer los elementos comunes y los diferentes. Algunas veces se debió recurrir a la entrevista informal debido a la heterogeneidad académica de las personas. A partir de ellas se realizó una matriz de análisis para cada grupo de actores.

Con los transportadores en estudio se utilizó un muestreo de juicio o criterio (ELORZA, 1994) considerando el "fenómeno de saturación" propuesto por OSORIO (1994).

Características de los vehículos: Se observaron las condiciones de diseño del vehículo (modelo, material del piso, tipo de cama, material del techo, división interna dela carrocería, bordes etc.), de acuerdo a los requerimientos sanitarios y de inocuidad en la producción primaria establecidos en la resolución colombiana ICA 002341 de 2007.
Tiempo de espera de los vehículos a la entrada del frigorífico: Para la estimación del tiempo de espera del vehículo cargado con vacunos en la entrada del frigorífico, se registró la hora exacta de llegada a la portería y la hora de ingreso (momento en que el vehículo pasó la puerta de entrada) de 34 viajes de ganado. En el lapso de espera, la empresa verificó que la documentación presentada por el transportista.

\section{Tiempo de espera de los vehículos en frigorífico} previo al descargue: El tiempo de espera en frigorífico previo al descargue se calculó a partir de los registros de la hora exacta de llegada de 35 viajes de ganado a la zona de descargue y la hora exacta en que se abrió la puerta del camión para la salida de los animales.

Tiempo de desembarque: La medición del tiempo de desembarque de los bovinos se realizó desde el momento de la apertura de la puerta del vehículo hasta la salida del último animal, usando un cronómetro.

Densidad de carga: El procedimiento para calcular la densidad de carga consistió en medir con una cinta métrica los lados del vehículo para calcular el área interna del mismo. Posteriormente, se tomaron los pesos individuales de los semovientes, mediante el pesaje en una báscula electrónica (PROGRAN 1500ss), inmediatamente después del descargue. Conocida el área del camión, el peso y número de animales transportados se procedió a calcular la densidad de carga $(\mathrm{kg}$ de peso vivo/m²) según AGUAYO (2006).

Estimación de la distancia recorrida: Conociendo la ubicación de las fincas (dato que fue averiguado mediante la entrevista semiestructurada aplicada a los transportistas), se hizo una estimación de la distancia recorrida en km, utilizando la herramienta de Google Maps.

Determinación de la heterogeneidad del lote a través del peso de los animales por viaje realizado: Para la determinar el grado de heterogeneidad del peso de los bovinos transportados en cada viaje se registraron los pesos individuales de los animales usando el programa estadístico Info Stat v.e (Di RENZO et al., 2011) y se procedió al cálculo de la desviación estándar, la cual se usó como medida de dispersión 
de los datos. Además de la desviación estándar, usando el mismo programa, se obtuvieron los valores referentes a: número de bovinos por viaje, promedio de peso, peso máximo y mínimo de los animales por cada viaje. A través de estos últimos valores se analizó la variabilidad del lote.

Análisis de la información: Para el análisis estadístico de la información se aplicó estadística descriptiva y se realizaron tablas de frecuencia para cada una de las variables estudiadas, usando el programa estadístico InfoStat v.e (DI RENZO et al., 2011). Posterior a la agrupación y análisis de los datos de transporte correspondientes al presente trabajo, se correlacionaron con los datos obtenidos en otros estudios paralelos al mismo y realizados a nivel de corrales de espera y en la línea de sacrificio de la misma planta de beneficio. Se evaluaron nueve lotes en común entre estas tres instancias, con un total de 131 animales. Las variables correlacionadas para los 131 animales fueron: Distancia recorrida, densidad de carga, tiempo que los camiones esperaron tanto a la entrada como al interior del frigorífico y tiempo de descarga de los animales, correspondientes a la etapa de transporte; presencia de hematomas y $\mathrm{pH}$ de la canal en frio (esta variable fue tomada en 50 animales de los 131 evaluados), las cuales se evaluaron en la línea de sacrificio, y variables comportamentales de los animales en corrales (confrontaciones, defecación, orina, montas y reactividad). En la parte referente a resultados se hará énfasis solamente a las correlaciones significativas $(P<0.05)$. El análisis de correlación utilizado fue el de Pearson, utilizando el software InfoStat v.e (DI RENZO et al., 2011). Las variables correlacionadas se escogieron considerando el posible efecto del transporte de los animales sobre su comportamiento posterior al descargue, en los corrales de espera, y sobre aspectos relacionados con la canal.

Para estudiar el posible efecto del transporte sobre la presencia de hematomas, verificadas en las canales, se aplicó un análisis de regresión logística, haciendo énfasis en aquellas variables de transporte que incidieron directamente sobre la presencia de hematomas en el tejido del animal. El análisis se realizó utilizando la función "Regresión Logística" para datos categorizados, del Programa InfoStat v.e (DI RENZO et al., 2011). Se planteó el análisis para variables de tipo dicotómico en relación a una o más variables independientes regresoras. Los valores que se asumieron fueron SI o NO (presencia o ausencia de hematomas), como 1 o 0 , respectivamente. Se modeló la transformación Logit de la probabilidad de éxito como una función lineal de las variables consideradas (DI RENZO et al., 2011). Las variables del modelo se presentan con los coeficientes de regresión, el error estándar, la estimación de la razón de productos cruzados (odd ratios), el intervalo de confianza, -2(Lo-L1) y el valor de $p$ para la prueba de hipótesis: Ho: $\beta i=0$ versus $\beta i \neq 0$, realizada usando el estadístico Chi Cuadrado con 1 grado de libertad.

\section{Resultados}

Estudio etnográfico. Los resultados referentes a variables cualitativas del estudio etnográfico se presentan en la Tabla 1.

Tabla 1. Características demográficas y actitudes de los conductores de ganado vacuno referentes al bienestar animal (BA) durante la etapa de transporte.

\begin{tabular}{|c|c|c|c|c|c|}
\hline Variable & $\mathbf{n}$ & $\%$ & Variable & $\mathbf{n}$ & $\%$ \\
\hline Edad (años) & \multicolumn{5}{|c|}{$\begin{array}{c}\text { Capacitación sobre } \\
\text { BA }\end{array}$} \\
\hline $20-30$ & 1 & 5 & $\mathrm{Si}$ & 0 & 0 \\
\hline $31-40$ & 5 & 25 & No & 20 & 100 \\
\hline $41-50$ & 1 & 5 & & & \\
\hline$>50$ & 13 & 65 & & & \\
\hline $\begin{array}{l}\text { Experiencia en el } \\
\text { oficio (años) }\end{array}$ & \multicolumn{5}{|c|}{$\begin{array}{l}\text { Métodos usados } \\
\text { para levantar } \\
\text { animales }\end{array}$} \\
\hline $7-15$ & 6 & 33 & Tábano & 3 & 15 \\
\hline \multirow[t]{3}{*}{$20-40$} & 12 & 67 & Palo & 11 & 55 \\
\hline & & & Palo y tábano & 5 & 25 \\
\hline & & & Gasolina en los ojos & 1 & 5 \\
\hline
\end{tabular}

n=número de transportistas

La mayoría de los conductores (83\%) manifestaron que realizan el oficio por necesidad, principalmente, porque no tienen otra fuente de empleo y deben llevar el sustento a sus familias, solo la minoría de esta población (17\%) realizan la actividad porque les motiva y es de su agrado. En cuanto al nivel de escolaridad, el $68 \%$ de los conductores encuestados manifestaron haber terminado sus estudios secundarios. Los que solo realizaron estudios primarios resultaron ser personas mayores de 50 años. 
En aspectos de índole laboral, la mayoría de encuestados $(87 \%)$ manifestaron haber desempeñado trabajos como conductores de transporte público o siempre han estado en el oficio de manejo de camiones, solo muy pocos manifestaron que con anterioridad desempeñaron oficios relacionados con la ganadería.

A continuación se presenta un resumen sobre las opiniones de los transportista frente al oficio de conducción y manejo de los bovinos: En cuanto a la mejor forma de trabajar y transportar a los animales los transportistas consideran que para desempeñar este trabajo adecuadamente se debe primero que todo tener una buena cama sobre la cual se van a parar los animales, luego cargar el vehículo de tal manera que no queden los animales muy hacinados, hacer una buena carga y dividir la carrocería en partes, respetando la capacidad del carro, es decir no exceder la carga permitida. Conducir suave, a baja velocidad, trabajar con calma, no frenar bruscamente y si el trayecto del viaje se prolonga, se deben realizar paradas. Sin embargo, un conductor consideró que los mejores viajes se hacen cuando se conduce rápido y se llega rápido al sitio de descargue, este último fue uno de los conductores de mayor edad, y en el seguimiento que se le hizo, fue el transportador que cargaba el mayor número de animales en cada viaje.

Por otra parte, para el $45 \%$ de los conductores encuestados, es indiferente transportar el ganado en la mañana o en la tarde, el $55 \%$ consideran que el comercio del ganado se desarrolla durante las horas de la mañana, ya que en las horas de la tarde son muy pocos los productores que los contratan para movilizar los animales hasta la planta de beneficio.

La mayoría de los conductores (70\%) manifestaron que los animales adultos son más fáciles de transportar y los más difíciles son los terneros de levante, es decir, animales pequeños, ya que estos son animales más frágiles y además no mantienen el equilibrio dentro del camión, lo que causa inestabilidad del vehículo por caídas continuas. Afirman que hacen paradas durante el trayecto priorizando sus necesidades y luego monitorean las condiciones de los animales; ellos consideran que deben llegar rápido a donde van a recibir el ganado, para no perder el negocio; sin embargo, después de asegurar el viaje, se dedican a sus tareas personales como desayunar o almorzar, abastecer el vehículo con combustible dejando a un lado las necesidades de los animales.

Características generales de los vehículos. En la Tabla 2 se presentan los datos referentes a las características generales de los vehículos. En cuanto a la capacidad de carga de los camiones, según opinión de los conductores, esta es muy bien manejada cuando se transporta carga muerta, sin embargo, todos los conductores manifestaron que cuando se transporta carga viva (bovinos) son muchos los factores que influyen en la densidad, como el tipo de animal, el peso y la edad de los mismos.

Tabla 2. Uso, características estructurales, modelo y capacidad de carga de los vehículos utilizados para transporte de bovinos hacia planta de faenado

\begin{tabular}{cccccc}
\hline Variable & $\mathbf{n} \%$ & Variable & $\mathbf{n}$ & $\%$ \\
\hline $\begin{array}{c}\text { Uso exclusivo } \\
\text { transporte bovino }\end{array}$ & \multicolumn{5}{c}{ Piso antideslizante } \\
$\mathrm{Si}$ & 9 & 45 & $\mathrm{Si}$ & 14 & 70 \\
$\mathrm{No}$ & 11 & 55 & $\mathrm{No}$ & 6 & 30 \\
\hline Uso de cama & \multicolumn{5}{c}{ Material del piso } \\
$\mathrm{Si}$ & 20 & 100 & Madera & 20 & 100 \\
No & 0 & 0 & Otro & 0 & 0 \\
\hline Material de la cama & \multicolumn{5}{c}{ Divisiones internas } \\
Viruta & 20 & 100 & Si & 14 & 70 \\
Otro & 0 & 0 & No & 6 & 30 \\
\hline Reciclaje de cama & \multicolumn{5}{c}{ Material de divisiones } \\
Si & 20 & 100 & Madera & 20 & 100 \\
No & 0 & 0 & Otro & 0 & 0 \\
\hline Uso de carpa & \multicolumn{7}{c}{ Bordes carrocería } \\
Si & 20 & 100 & Agudos & 20 & 100 \\
No & 0 & 0 & Curvos & 0 & 0 \\
\hline Capacidad de & \multicolumn{5}{c}{ Antigüedad del } \\
carga (t) & \multicolumn{5}{c}{ vehículo (años) } \\
3-9 & 9 & 45 & $7-14$ & 4 & 20 \\
10-17 & 11 & 55 & $25-65$ & 16 & 80 \\
\hline
\end{tabular}

$\mathrm{n}=$ número de transportistas

Tiempo de espera de los vehículos a la entrada del frigorífico. Los tiempos de espera a la entrada de la planta de beneficio para cada tipo de vehículo (grande y pequeño) se muestran en la Tabla 3. El $97 \%$ de los vehículos esperaron como máximo 12 minutos para ingresar al frigorífico, solo uno $(3 \%)$ superó este tiempo. 
Tabla 3. Valores medios del tiempo de espera de los vehículos a la entrada del frigorífico.

\begin{tabular}{|c|c|c|c|c|c|c|}
\hline \multirow{2}{*}{$\begin{array}{l}\text { Tipo de } \\
\text { vehículo }\end{array}$} & \multicolumn{2}{|c|}{$\begin{array}{l}\text { Viajes } \\
\text { realizados }\end{array}$} & \multicolumn{4}{|c|}{ Tiempo en portería (minuto) } \\
\hline & $\mathbf{n}$ & $\%$ & Promedio & DE & $\mathbf{R a}$ & ngo \\
\hline $\begin{array}{l}\text { Camión } \\
\text { grande }^{1}\end{array}$ & 20 & 58,8 & 3,56 & 1,72 & 0,5 & 7,0 \\
\hline $\begin{array}{l}\text { Camión } \\
\text { pequeño }\end{array}$ & 14 & 41,2 & 7,51 & 14,31 & 1,7 & 57 \\
\hline Total & 34 & 100 & 5,5 & 8,0 & 1,1 & 32,0 \\
\hline
\end{tabular}

Tiempo de espera de los vehículos dentro de la planta de beneficio previo al desembarque. En la Tabla 4 se muestran los tiempos de espera dentro de la planta de beneficio para cada tipo de vehículo.

Tabla 4. Valores medios del tiempo de espera de los vehículos dentro de frigorífico.

\begin{tabular}{cccccccc}
\hline \multirow{2}{*}{$\begin{array}{c}\text { Tipo de } \\
\text { vehículo }\end{array}$} & \multicolumn{2}{c}{$\begin{array}{c}\text { Viajes } \\
\text { realizados }\end{array}$} & \multicolumn{5}{c}{$\begin{array}{c}\text { Tiempo en frigorífico } \\
\text { (minuto) }\end{array}$} \\
\cline { 2 - 7 } & $\mathbf{n}$ & $\%$ & Promedio & DE & Rango \\
\hline $\begin{array}{c}\text { Camión } \\
\text { grande }\end{array}$ & 16 & 45,7 & 7,66 & 12,35 & 1 & 47 \\
$\begin{array}{c}\text { Camión } \\
\text { pequeño }\end{array}$ & 19 & 54,3 & 1,53 & 2,29 & 1 & 11 \\
& & & & & & & \\
Total & 35 & 100 & 4,6 & 7,3 & 1 & 29
\end{tabular}

$\mathrm{n}=$ número total de viajes realizados por los dos tipos de vehículos; ${ }^{1}$ Camión con $17,5 \mathrm{~m}^{2}$ de área en la carrocería; ${ }^{2}$ Camión con $8,5 \mathrm{~m}^{2}$ de área en la carrocería ${ }^{2}$. $\mathrm{DE}=$ Desviación estándar.

En esta etapa se observaron animales cansados y caídos principalmente en vehículos que esperaron tiempos prolongados dentro del frigorífico antes de iniciar la actividad de descargue de los bovinos. El $89 \%$ de los vehículos permanecieron hasta 10 minutos estacionados dentro de la planta. Un solo vehículo (3\%) permaneció por más de 37 minutos en espera.
Tiempo de descargue de los animales. Los valores referentes al tiempo de descargue se pueden apreciar en la Tabla 5.

Tabla 5. Valores medios del tiempo de descargue en frigorífico.

\begin{tabular}{cccccccc}
\hline \multirow{2}{*}{$\begin{array}{c}\text { Tipo de } \\
\text { vehículo }\end{array}$} & \multicolumn{2}{c}{$\begin{array}{c}\text { Viajes } \\
\text { realizados }\end{array}$} & \multicolumn{4}{c}{$\begin{array}{c}\text { Tiempo en frigorífico } \\
\text { (minuto) }\end{array}$} \\
\cline { 2 - 7 } & $\mathbf{n}$ & $\%$ & Promedio & DE & Rango \\
\hline $\begin{array}{c}\text { Camión } \\
\text { grande }\end{array}$ & 34 & 57,6 & 3,36 & 1,92 & 1 & 10 \\
$\begin{array}{c}\text { Camión } \\
\text { pequeño }\end{array}$ & 25 & 42,4 & 2,24 & 1,16 & 1 & 6 \\
& & & & & & & \\
Total & 59 & 100 & 2,8 & 1,5 & 1 & 8 \\
\end{tabular}

$\mathrm{n}=$ número total de viajes realizados por los dos tipos de vehículos; ${ }^{1}$ Camión con $17,5 \mathrm{~m}^{2}$ de área en la carrocería; ${ }^{2}$ Camión con $8,5 \mathrm{~m}^{2}$ de área en la carrocería ${ }^{2}$. DE=Desviación estándar.

Durante la espera para el descargue dentro del frigorífico, se pudo observar que los transportistas utilizaron métodos dolorosos para lograr el descenso de los animales del vehículo, métodos que incluyeron el uso de palos, tablas y tubos de hierro. El $54 \%$ de los vehículos se descargaron en un lapso de tiempo entre 1 y 2,8 minutos, mientras que el $34 \%$ lo hicieron entre 2,8 y 4,6 minutos, el $12 \%$ restante invirtieron entre 4,6 y 10 minutos.

Densidad de carga. El $90 \%$ de los animales ingresados a la planta presentaron pesos superiores a $350 \mathrm{~kg}$. La densidad de carga registrada para cada viaje, según el tipo de vehículo, se muestra en las tablas 6 y 7 .

Tabla 6. Promedios y medidas de dispersión para el peso de los animales y densidad de carga de los viajes realizados por camiones grandes (área de 17,5 $\mathrm{m}^{2}$ aprox.)

\begin{tabular}{ccccccc}
\hline Variable & n & Media & DE & CV & Min & Max \\
\hline $\begin{array}{c}\text { Peso de animales } \\
(\mathrm{kg})\end{array}$ & 34 & 420,6 & 45,6 & 10,9 & 335,3 & 527,1 \\
$\begin{array}{c}\text { Densidad calculada } \\
\left(\mathrm{kg} / \mathrm{m}^{2}\right)\end{array}$ & 34 & 320,5 & 86,3 & 26,9 & 110 & 411,7 \\
$\begin{array}{c}\text { Espacio } \\
\left(\mathrm{m}^{2} / \text { animal }\right)\end{array}$ & 34 & 1,4 & 0,56 & 38,7 & 0,91 & 3,6 \\
\hline
\end{tabular}

$\mathrm{n}=$ representa el número de viajes realizados; $\mathrm{DE}=$ desviación estándar $\mathrm{CV}=$ coeficiente de variación; Min.=valor mínimo; Max.=valor máximo. 
Tabla 7. Promedios y medidas de dispersión para el peso de los animales y densidad de carga de los viajes realizados por camiones pequeños (área de 8,5 $\mathrm{m}^{2}$ aprox.)

\begin{tabular}{ccccccc}
\hline Variable & $\mathbf{n}$ & Media & DE & CV & Min & Max \\
\hline $\begin{array}{c}\text { Peso de animales } \\
(\mathrm{kg})\end{array}$ & 34 & 400,5 & 49,0 & 12,2 & 306,6 & 527,1 \\
$\begin{array}{c}\text { Densidad calculada } \\
\left(\mathrm{kg} / \mathrm{m}^{2}\right)\end{array}$ & 34 & 285,2 & 83,5 & 29,3 & 76,7 & 394,6 \\
$\begin{array}{c}\text { Espacio } \\
\left(\mathrm{m}^{2} / \text { animal }\right)\end{array}$ & 34 & 1,56 & 0,65 & 41,7 & 1,09 & 4,4 \\
\hline
\end{tabular}

$\mathrm{n}=$ número de viajes realizados; $\mathrm{DE}=$ desviación estándar; $\mathrm{CV}=$ coeficiente de variación; Min.=valor mínimo; Max.=valor máximo.

En términos generales, las densidades de animales en los vehículos grandes estuvieron más próximas a las recomendadas por el ICA (2007) en comparación a las observadas en los vehículos de menor capacidad, como se aprecia en las figuras 1 y 2 .

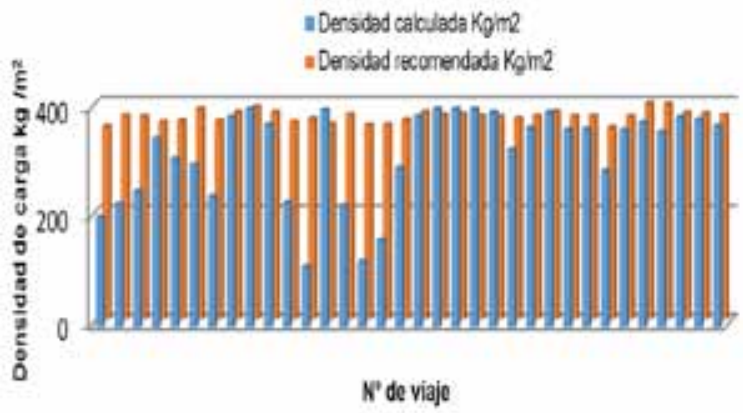

Figura 1. Densidades de carga calculadas para camiones grandes (aprox. 17,5 $\mathrm{m}^{2}$ ) comparadas con las recomendadas por el ICA (2007).

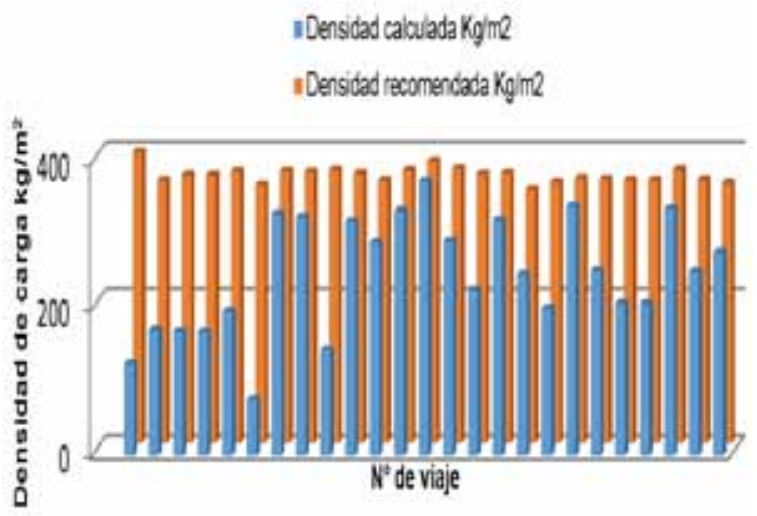

Figura 2. Densidades de carga calculadas para camiones pequeños (aprox. 8,5 $\mathrm{m}^{2}$ ) comparadas con las recomendadas por el ICA (2007).
Agrupando todos los viajes, la densidad promedio encontrada $\left(289,4 \mathrm{~kg} / \mathrm{m}^{2} ; \mathrm{DE}=90,24\right)$ estuvo por debajo de la recomendado por el ICA (2007), que sería de $368 \mathrm{~kg} / \mathrm{m}^{2}$, para animales de $400 \mathrm{~kg}$. En la Tabla 8 se presentan las frecuencias observadas para diferentes densidades de carga.

Tabla 8. Tabla de frecuencia para la densidad $\left(\mathrm{kg} / \mathrm{m}^{2}\right)$ de carga de los vehículos

\begin{tabular}{ccccc}
\hline Clase & LI & LS & FA & FR \\
\hline 1 & 76,7 & 143,7 & 5 & 0,08 \\
2 & 143,7 & 210,7 & 9 & 0,15 \\
3 & 210,7 & 277,7 & 9 & 0,15 \\
4 & 277,7 & 344,7 & 15 & 0,25 \\
5 & 344,7 & 411,7 & 21 & 0,36 \\
\hline
\end{tabular}

$\mathrm{LI}=$ Límite inferior; $\mathrm{LS}=\mathrm{Li}$ imite superior; $\mathrm{FA}=$ frecuencia absoluta; $F R=F$ recuencia relativa.

El $36 \%$ de los vehículos viajaron con densidad entre 344,7 y $411,7 \mathrm{~kg} / \mathrm{m}^{2}$, seguido por el rango de 277,7 a $344,7 \mathrm{~kg} / \mathrm{m}^{2}(25 \%)$. El $38 \%$ de los viajes se realizaron con densidades inferiores a $277,7 \mathrm{~kg} / \mathrm{m}^{2}$.

Distancia recorrida desde la finca o lugar de origen hasta frigorífico. Las distancias recorridas por los vehículos se indican en la Tabla 9. Se observó que los camiones más grandes recorrieron una mayor distancia en comparación a los más pequeños.

Tabla 9. Distancia recorrida $(\mathrm{km})$ por los diferentes tipos vehículos

\begin{tabular}{cccccccc}
\multirow{2}{*}{$\begin{array}{c}\text { Tipo de } \\
\text { vehículo }\end{array}$} & \multicolumn{3}{c}{$\begin{array}{c}\text { Viajes } \\
\text { realizados }\end{array}$} & \multicolumn{4}{c}{ Distancia recorrida (Km) } \\
\cline { 2 - 7 } & $\mathbf{n}$ & $\%$ & Prom. & DE & Rango \\
\cline { 2 - 7 } $\begin{array}{c}\text { Camión } \\
\text { Grande }\end{array}$ & 34 & 57,6 & 34,1 & 35,45 & 5,1 & 176 \\
$\begin{array}{c}\text { Camión } \\
\text { Pequeño }\end{array}$ & 25 & 42,4 & 9,02 & 14,21 & 5,1 & 73,8 \\
Total & 59 & 100 & 21,6 & 24,8 & 5,1 & 124,9
\end{tabular}

$\mathrm{n}=$ número de viajes realizados por los dos tipos de vehículos; ${ }^{1}$ Camiones con $17,5 \mathrm{~m}^{2}$ de área en la carrocería; ${ }^{2}$ Camión con $8,5 \mathrm{~m}^{2}$ de área en la carrocería; Prom. $=$ promedio; $\mathrm{DE}=$ Desviación estándar.

Los viajes que se analizaron durante el periodo de estudio para determinar la distancia recorrida por los vehículos, procedieron de 16 localidades diferentes. El $85 \%$ de los viajes analizados recorrieron distancias inferiores a $39,3 \mathrm{~km}$; el 
$10,5 \%$ entre 39,2 y $73,5 \mathrm{~km}$, y el resto $(6 \%)$ distancias superiores, con un valor máximo de $176 \mathrm{~km}$.

Relación entre variables referentes al transporte con variables de la línea de sacrificio. La distancia recorrida $(P=0,0163$; Est. $=-0,005)$, la densidad de transporte $(P=0,0033$; Est. $=-0,11)$ y el tiempo invertido en el desembarque $(P=0,0002$; Est. $=0,0054)$ de los animales fueron variables predictoras de la presencia de hematomas. Bajo las condiciones del estudio, los recorridos cortos con densidades de carga bajas incrementaron la probabilidad de presencia de hematomas en la canal, al igual que tiempos de descargue prolongados. Correlaciones significativas $(P<0,05)$ se observaron entre la distancia recorrida y el número de hematomas $(r=0,20)$, el $\mathrm{pH}$ de la canal fría $(r=0,80)$ y la densidad de carga $(r=0,47)$; entre el tiempo de descargue y el número de hematomas $(r=0,25)$ y el $\mathrm{pH}$ de la canal fría $(r=0,84)$; entre la densidad de carga y el número de hematomas $(r=-0,20)$; entre el tiempo de espera en frigorífico y el número de hematomas $(r=0,35)$ y el $\mathrm{pH}$ de la canal fría $(r=0,73)$.

\section{Discusión}

En el presente estudio los transportadores no habían recibido capacitación sobre BA, lo que se constituye en una debilidad que debe ser solucionada. ROMERO et al. (2011) encontraron también cifras bajas en materia de capacitación, en una región de Colombia, ya que solamente el $4,1 \%$ de los conductores habían recibido algún tipo de capacitación, lo cual evidencia la falta de conocimiento que tienen los transportadores del país en el manejo de bovinos. Los programas de capacitación sobre temas de BA al personal encargado del manejo de bovinos en la etapa de transporte son esenciales para que las normas establecidas con respecto al tema sean consistentes (SCHWARTZKOPF-GENSWEIN el al., 2008). Según GALLO (2009), una conducción adecuada y prudente, evitando maniobras bruscas en la vía, favorece el bienestar de los animales y permite que lleguen en buenas condiciones al lugar de sacrificio. Además, GRANDIN (2003) indica que si a los transportistas se les incentiva con dinero, estos van a brindar un mejor trato a los bovinos y de esta manera reducir las diferentes contusiones y mermas de peso, por lo que esta recomendación debería ser complemento de la capacitación. En este sentido, a partir de la recomendación, se podría aumentar el grado de satisfacción al realizar este tipo de trabajo, y mejorar la percepción de los transportadores sobre el oficio realizado, ya que en el caso específico del estudio realizado, el $83 \%$ lo realizan por pura necesidad.

En cuanto al uso de diversos métodos para levantar animales caídos, se encontró que es muy usual el empleo de la fuerza y de herramientas u objetos que causan daños o miedo en el animal. En Colombia, ROMERO et al. (2011) verificaron el uso continuo de tábano $(46 \%)$ o picana eléctrica en el transporte de ganado vacuno y otro tipo de objetos contundentes que pueden aumentar la reactividad del animal y disminuir su bienestar. Además, GRANDIN (2000b) plantea que si la picana eléctrica se usa de manera continua en vacunos de raza cebú se les puede causar la muerte. De igual manera, el uso de ésta, acompañada con palos y otros objetos contundentes, produce daños en las canales y pieles, generando pérdidas económicas debido a los decomisos efectuados en áreas lesionadas (TAFUR y ACOSTA, 2006). La aplicación de estas herramientas se evidencia en las canales en forma de hemorragias petequiales y lesiones de distinta forma, profundidad y tamaño, y son un reflejo claro del mal trato que han recibido los animales, y un pobre BA (GALLO, 2008c). Estudios realizados en otras regiones de Colombia, por ROMERO et al. (2011), demuestran que tampoco se tienen en cuenta criterios de BA, ya que los autores encontraron que los transportadores utilizan de igual manera métodos dolorosos para levantar animales caídos, un $46,8 \%$ utilizan tábano, mientras que un $16,1 \%$ utilizan palos y varetas de madera. La OIE (2015) propone que el tábano solo debe de ser usado en condiciones extremas y no de manera repetitiva para lograr que los animales se levanten, y en el caso de usarlo, no debe superar los $30 \mathrm{v}$ de electricidad ni ser colocado en partes como ojos, orejas, vientre $u$ órganos genitales.

La legislación Colombiana, en la Resolución ICA 002341 de 2007, estableció normas específicas relacionadas con el transporte, en las que se incluyen los requisitos que deben cumplir los conductores. En términos generales los vehículos 
evaluados en el estudio se ajustan a la normatividad con respecto a su conformación. Sin embargo, se destacó la presencia de bordes agudos en todos los vehículos, lo que puede causar lesiones en los animales. El uso de cama es común, pero el uso de la misma, hasta por seis o siete días dificulta la limpieza y desinfección de los vehículos y podría tener consecuencias negativas de tipo sanitario. El cambio de cama se debe hacer luego de cada viaje, según recomendaciones de la OIE (2015) y de DE LA SOTA (2005).

Otro aspecto de particular importancia es que los vehículos (55\% de la población estudiada) no son usados exclusivamente para el transporte de vacunos, sino que su uso se amplía a cualquier otro tipo de carga. Resultados similares encontraron ROMERO et al. (2011), en donde el $79.5 \%$ de los conductores encuestados, además de bovinos transportaban otras especies animales y carga muerta o cualquier tipo de mercancía. Lo anterior indica que el transporte en el país no es especializado, debido a que se transportan otro tipo de mercancías en vehículos para transporte bovino (ROMERO et al., 2012b). Según la legislación colombiana el camión solo debe ser utilizado para el transporte exclusivo de bovinos después de haberse lavado y desinfectado previo a cada carga, por lo que este hallazgo se puede considerar como uno de los puntos a ser solucionados.

En cuanto a la densidad de carga, en la mayoría de los viajes, tanto camiones grandes como pequeños se presentaron densidades de carga bajas con respecto a la establecida por el ICA. De la misma manera, GALLO et al. (2005) encontraron diferencias significativas en cuanto a las densidades de carga; esta fue mayor en novillos $\left(463 \mathrm{~kg} / \mathrm{m}^{2}\right)$ en contraste con otra clase de bovinos, por lo que son estos los animales que más se benefician y los de mayor valor económico. En Colombia, ROMERO et al. (2012b) realizaron un estudio con 2288 bovinos procedentes de 275 fincas, localizadas en 13 Departamentos de Colombia, y encontraron densidades de carga entre los 300 y $350 \mathrm{~kg} / \mathrm{m}^{2}$ las cuales resultaron estar por debajo de la recomendación para Colombia. Concluyeron además que esto influyó de manera indirecta sobre la presencia de contusiones, es decir, a menores densidades de carga mayor presencia de contusiones, como se observó también en el presente estudio. Caso contrario encontró GALLO et al. (2005) en dos plantas faenadoras de Chile, en donde observaron densidades de carga próximas a los $457 \mathrm{~kg} /$ $\mathrm{m}^{2}$ que resultan ser relativamente altas si se aplica la ecuación planteada por la Farm Animal Welfare Council (1991) y las cuales aumentaron la incidencia de hematomas. De estos estudios se puede afirmar que tanto a densidades de carga altas como bajas se afecta el bienestar de los animales y la calidad de la carne debido a las presencia de contusiones de cualquier tipo (GALLO, 2008c).

El tiempo promedio de espera previo al desembarque de animales, tanto fuera como dentro de las instalaciones de la planta, tuvo una duración de 5,5 y 4,6 minutos, respectivamente, lo que se considera como tiempos relativamente cortos. Sin embargo, se presentaron casos hasta de 57 y 47 minutos a la entrada y dentro del frigorífico, respectivamente, considerados como tiempos demasiado extensos. Se recomienda un tiempo de hasta 15 minutos desde la llegada hasta el desembarque de los animales, y no sobrepasar nunca los 20 minutos, para evitar estrés y agotamiento (GRANDIN, 2003; HUERTAS, 2009). En este caso se recomienda continuar mejorando, evitando que existan casos de esperas superiores a los 20 minutos. En este caso, se aclara que en ocasiones los tiempos de espera prolongados no se deben a fallas por parte de la planta, sino que se pueden presentar problemas con la documentación referente al viaje de ganado.

El tiempo de descargue, en términos generales, se ubicó en rangos considerados aceptables (2,8 min.). HUERTAS y RODRIGUEZ (2008) encontraron un tiempo promedio en la descarga de 5,4 (D.E. $\pm 0,2$ ) minutos, posiblemente porque transportaban mayor número de animales y en vehículos de mayor tamaño. En Colombia, ROMERO et al. (2013) encontraron tiempos de descargue de 4,52 (D.E. $\pm 0,8$ ) minutos, los cuales resultan ser cortos según MARIA et al. (2004), ya que están por debajo de los 15 minutos. Estos tiempos cortos en la descarga pueden estar relacionados con el comportamiento de fuga del bovino, debido a que la planta de beneficio les resulta ser un ambiente totalmente nuevo y sumado a ello los operarios utilizan diferentes métodos para lograr el descenso de los bovinos del camión (ROMERO et al., 2013). 
La FAO (2004) y DE LASOTA (2005) recomiendan no mezclar animales de diferente procedencia $y$ tampoco de pesos diferentes en un mismo vehículo. En el presente estudio se evidenció heterogeneidad en cuanto al peso de los animales en algunos de los viajes analizados. Por ejemplo, en uno de los viajes se encontraron animales de 287 hasta $598 \mathrm{~kg}$ de peso viajando simultáneamente en un mismo vehículo de porte pequeño, y en otro caso, animales con peso de 239 hasta $640 \mathrm{~kg}$ en un vehículo de porte mayor. Este tipo de manejo no es el más adecuado, debido a que se van a crear comportamientos de jerarquía entre los animales, generándose así, peleas y conflictos entre ellos afectando su bienestar (LUDTKE et al., 2012). Además de esto, los enfrentamientos o peleas durante el transporte pueden generar contusiones y hematomas en la canal comprometiendo su calidad (GALLO, 2009). Aunque no se observó este problema del bienestar animal en todos los vehículos, es un aspecto que se debe vigilar, de manera que se garanticen aspectos ligados al BA, como también a la calidad e inocuidad de la carne.

Según los análisis de correlación y de regresión logística se pudo evidenciar que de una u otra forma el BA y la calidad de la carne son dependientes de las condiciones del transporte y el manejo dado a los animales durante el mismo. En este estudio, la distancia recorrida por el vehículo provocó la alteración del $\mathrm{pH}$, pero no insidió sobre presencia de hematomas en la canal, es decir, estos últimos no dependieron de que tan cerca o tan lejos viniera el camión. Los hematomas dependieron de la densidad de carga con la cual viajaron los camiones. Los camiones que viajaron a menor distancia lo hicieron con densidades de carga bajas, aumentando así el riesgo de que se generen mayor número de hematomas. Las distancias recorridas por los camiones en este estudio no fueron lo suficientemente largas como para que se generaran contusiones o lesiones en los animales. GUIDOBONO (2014) considera que las lesiones o hematomas en las canales se generan cuando los camiones recorren distancias superiores $(250-600 \mathrm{~km})$. En este estudio las distancias recorridas no superaron los $200 \mathrm{~km}$.

Los valores elevados de $\mathrm{pH}$ altos en la canal post-morten a $36 \mathrm{~h}$, debidos a mayores distancias recorridas, podrían estar relacionados con las precarias condiciones de las vías terciarias y no con la distancia como tal. Según GALLO (2009), las vías en mal estado son uno de los factores causantes de estrés en los animales. En el descargue, estos problemas pudieron deberse más al estrés que experimentaron los bovinos durante este evento que al tiempo empleado durante el mismo, ya que según MARIA et al. (2004) y NEVILLE (1998), el desembarque de los animales en el frigorífico puede ser mucho más estresante que el transporte propiamente dicho. Además, los tiempos registrados no superaron el límite permitido. De igual manera, KNOWLES (1999) afirma que el desembarque es unos de los eventos causantes de lesiones y hematomas en el tejido del bovino. Los valores elevados de $\mathrm{pH}$ en la canal se deben al consumo rápido de glucógeno que experimentan los animales al momento de ser descargados y en los momentos previos al sacrificio por causa del estrés, lo cual no permite el descenso normal del pH (FAO, 2001; GALLO, 2008b).

\section{Conclusiones}

Se evidenciaron fallas que repercuten negativamente en el bienestar de vacunos durante el transporte y descargue en la planta de faenado y sobre la calidad de la canal, aunado a la falta de capacitación de los conductores en el tema del BA. Densidades de carga por debajo y por encima de lo recomendado, heterogeneidad en el peso animal en un mismo viaje y el uso de objetos no adecuados para levantar animales caídos, entre otros, son problemas que deben ser solucionados mediante la capacitación y aplicación de protocolos de BA adaptados a las condiciones del estudio. 


\section{Referencias}

AGUAYO, L. 2006. Características del transporte de bovinos desde puerto Chacabuco (xi región) hasta puerto MONTT ( $x^{a}$ región). (Trabajo de grado médico veterinario). Universidad Austral de chile. Facultad de ciencias veterinarias Valdivia - Chile: 2006. Disponible en: URL: http://intranet.uach.cl/dw/canales/ repositorio/archivos/1995.pdf

ALAMINOS, A. 2005. El análisis de la realidad social. Modelos estructurales de covarianza. Observatorio Europeo de Tendencias sociales. Universidad de Alicante. Valencia España 2005. Disponible en: URL: http://www.obets.ua.es/obets/libros/modelos.pdf

BROOM, D. M. 1986. Indicators of poor welfare. British Veterinary Journal 142 (6), 524. Disponible en: URL: http://endcap.eu/wp-content/uploads/2015/06/Broom-1986-Indicators-of-poor-animal-welfare.pdf

BROOM, D. M. 1991. Animal welfare: concepts and measurement. Journal of Animal Science 69(10): 4167-4175. Disponible en: URL: http://www.infozoos.org/descargas/AnimalWelfare.pdf

DE LA SOTA, M. 2005. Manual de procedimiento en el transporte de animales. Dirección Nacional de Sanidad Animal (SENASA) 3:19. Disponible en: URL: http://www.produccion-animal.com.ar/legales/19manual_transporte.pdf

DE LUQUE-F, A.; DUSSAN, C, D. 2009. Evaluación del bienestar de bovinos mediante la identificación de lesiones traumáticas macroscópicas presentes en la canal. Trabajo de grado de medicina veterinaria. Universidad de la Salle. Facultad de Medicina Veterinaria. Santa Fe de Bogotá 2009. Disponible en: URL: http://repository.lasalle.edu.co/bitstream/handle/10185/6001/T14.09\%20L974e.pdf?sequence=1

DI RENZO-J. A.; CASANOVES F.; BALZARINI-M. G.; GONZÁLEZ, L.; TABLADO, M.; ROBLEDO-C. W. 2011. InfoStat versión 2011e. Grupo InfoStat, FCA Universidad Nacional de Córdoba, Argentina. Disponible en: URL: http://www.infostat.com.ar

FAO. 2001. Directrices para el manejo, transporte y sacrificio humanitario del ganado: transporte del ganado. Capítulo 6 [en línea]. 2001 [citado en junio del 2016] 6. Disponible en: URL: http://www.fao.org/ docrep/005/x6909S/x6909s00.htm\#Contents

FAO. 2004. Transporte de animales al sacrificio. Sección 5 [en línea], 2014 [citado en junio del 2016] 5: 3-14. Disponible en: URL: ftp://ftp.fao.org/docrep/fao/010/y5454s/y5454s05.pdf

FARM ANIMAL WELFARE COUNCIL. 1991. Report on the European Commission. The welfare of animals during transport (details for horses, pigs, sheep and cattle). Report of the Scientific Committee on Animal Health and Animal Welfare Adopted. On 11 March 2002. Recuperado de http://www.dyrenesbeskyttelse. $\underline{\mathrm{dk} / \text { sites/default/files/landbrugsdyr/out71_en.pdf }}$

GALLO, C. 2009. Transporte y reposo pre-sacrificio en bovinos y su relación con la calidad de la carne. Bienestar animal y calidad de la carne. (EDS.) MOTA, R.; GUERRERO, I. BM Editores. México. p.15-36. Disponible en: URL: http://intranet.uach.cl/dw/canales/repositorio/archivos/28/4121.pdf

GALLO, C. 2008a. Using scientific evidence to inform public policy on the long distance transportation of animals in South America. Veterinaria Italiana 44(1): 113-120. Disponible en: URL: http://www.izs.it/ vet italiana/2008/113 120.pdf

GALLO, C. 2008b. Transporte e bem-estar animal. Ciênc. Vet. Tróp 11(1):70-79. 
GALLO, C.; WARRISS, P.; KNOWLES, T.; NEGRÓN, T.; VALDES, A.; MENCARINI, I. 2005. Densidades de carga utilizadas para el transporte de bovinos destinados a matadero en Chile. Arch. Med. Vet 37(2): 155-158. Disponible en: URL: http://mingaonline.uach.cl/pdf/amv/v37n2/art10.pdf

GALLO, C.; TADICH, N. Bienestar animal y calidad de carne durante los manejos previos al faenamiento en bovinos. Revista Electrónica de Veterinaria [en línea]. Octubre, 2008 [citado el 5 mayo del 2016] 9(10B). Disponible en: URL: http://www.redalyc.org/articulo.oa?id=63617111001

GRANDIN, T. 2000. Principios de comportamiento animal para el manejo de bovinos y otros herbívoros en condiciones extensivas. En: Grandin, T (comp.) Livestock handling and transport. Cabi Publishing, Wallingford, Oxon (reino unido) 5: (63-85). Disponible en URL: http://www.bienestaranimal.org.uy/files/ docs_grandin_traducidos.pdf

GRANDIN, T. 2003. El transporte del ganado: guía para las plantas de faena. Depto. De Ciencia animal Colorado state University, fort collins, EE.UU. Sitio Argentino de Producción Animal, Traducción: DR. Marcos Giménez-Zapiola [en línea], 2003 [citado en junio del 2016]. PP 6. Disponible en URL: http:// www.produccionanimal.com.ar/etologia y bienestar/bienestar en bovinos/70-transporte ganado para plantas faena.pdf

GRANDIN, T. 2010. Recommended animal handling guidelines \& audit guide: a systematic approach to animal welfare. Published by: American meat institute. (2010). June 2010 edition. With American Meat Institute Animal Welfare Committee certified and accredited by the professional animal auditors certification association. Disponible en: URL: http://www.animalhandling.org/ht/a/GetDocumentAction/i/63215

GUIDOBONO, M. 2014. Estudio de la evolución del porcentaje de contusiones en reses faenadas en una planta de Uruguay y su relación con la distancia recorrida por los animales como indicador de Bienestar Animal. Tesis de grado presentada como uno de los requisitos para obtener el título de Doctor en Ciencias Veterinarias. Universidad de la República Facultad de Veterinaria. Montevideo Uruguay. Disponible en: URL: http://www.fvet.edu.uy/index.php/ensenanza/educacion-continua/123-tesis-de-lafacultad-de-veterinaria/352-tesis-de-grado-2014

HUERTAS, S. 2009. Buenas prácticas de manejo durante el embarque y transporte a la planta de sacrificio. En: bienestar animal y calidad de la carne. MOTA-ROJAS, D.; GERRERO-LEGARRETA, I.; TRUJILLO-ORTEGA, M.E. Eds. Editorial BM Editores. México. p.73-84. Disponible en: URL: http://www. bienestaranimal.org.uy/files/04 BP\%20Manejo\%20embarque\%20y\%20transporte.pdf

HUERTAS, S.; GIL-R. A. Caracterización del transporte terrestre de bovinos hacia plantas de faena en Uruguay. Revista electrónica de Veterinaria [en línea]. Octubre, 2008 [citado en mayo del 2016] 9(10b): 1-14. Disponible en: URL: http://www.fao.org/fileadmin/user_upload/animalwelfare/BA047.pdf

ICA. Resolución No. 002341 (23 de agosto de 2007). Por la cual se reglamentan las condiciones sanitarias y de inocuidad en la producción primaria de ganado bovino y bufalino destinado al sacrificio para consumo humano. Bogotá D.C. Disponible en: URL: http://www.ica.gov.co/getattachment/0b5de556-cb4a-43a8a27a-cd9a2064b1ab/2341.aspx

KNOWLES, T. 1999. A review of the road transport of cattle. Veterinary Record 144: 197-201. Disponible en: URL https://www.researchgate.net/publication/13194535 A review of the road transport of cattle

LOZANO, F. 2008. "Hermenéutica activa. Los pretextos epistemológicos”. (Manuscritos), 17p. 
LUDTKE, C.; PANIM, J.; DANDIN, T.; CRUZ, P.; ANDRADE, J.; FERRARINI, C. 2012. Abate humanitário de bovinos. Wspa brasil - Sociedade Mundial de Proteção Animal. RJ, Brasil [en línea] 2012 [citado en junio del 2016]. Disponible en: URL: http://www.agricultura.gov.br/arq_editor/Manual\%20Bovinos.pdf

MARÍA, G.A.; VILLARROEL, M.; CHACÓN, G.; GEBRESENBET, G. 2004. Scoring system for evaluating the stress to cattle of commercial loading and unloading. Veterinary Record 154(26):818-821. Disponible en URL: https://www.researchgate.net/publication/8450074_Scoring_system_for_evaluating the_stress to cattle of commercial loading and unloadind

NEVILLE, G. 1998. Animal welfare and meat science. New York's. CABI Publishing 10 E40th Street. ISBN 0-85199-296-X (alk. paper).

ORGANIZACIÓN MUNDIAL DE SANIDAD ANIMAL - OIE. 2010. Código sanitario para los animales terrestres. Introducción a las recomendaciones para el bienestar de los animales. Editorial París (OIE), París. Disponible en: URL: https://www.oie.int/doc/ged/D7599.PDF

ORGANIZACIÓN MUNDIAL DE SANIDAD ANIMAL - OIE. Código sanitario para los animales terrestres. Capítulo 7.3, transporte de animales por vía terrestre. Revista electrónica OIE [en línea]. Septiembre del 2015 [citado en mayo del 2016] Artículo 7.3.2. Disponible en: URL: http://www.oie.int/index. php?id=169\&L=2\&htmfile=chapitre aw land transpt.htm

OSORIO, P.; FLOR, E. 1994. Las historias de Vida como técnica de investigación cualitativa. Serie Documentos de Trabajo. Pontificia Universidad Javeriana. Maestría en Desarrollo Rural. Santa Fe de Bogotá.

PÉREZ, H. 1994. Estadística para las ciencias sociales y del comportamiento". Editorial Oxford, segunda edición, Ciudad de México.

ROMERO, M.; GUTIÉRREZ, C.; SÁNCHEZ, J. 2012. Evaluación de contusiones como un indicador de bienestar animal durante el pre-sacrificio de bovinos. Revista Colombiana de Ciencias Pecuaria 25(2): 267-275. Disponible en: URL: http://www.scielo.org.co/pdf/rccp/v25n2/v25n2a12.pdf

ROMERO, M.; COBO, C.; GONZALES, L. 2013. Estudio de indicadores conductuales para evaluar el descargue de bovinos en plantas de beneficio. Revista Luna Azul 37: 12. Disponible en URL: http://www. redalyc.org/articulo.oa?id=321729206002

ROMERO, M.; SÁNCHEZ, J.; GUTIÉRREZ, C. 2011. Evaluación de prácticas de bienestar animal durante el transporte de bovinos para sacrificio. Rev. Salud pública 13(4): 7. Disponible en: URL: http://www. scielosp.org/pdf/rsap/v13n4/v13n4a13.pdf

ROMERO, P.; SÁNCHEZ, V. 2012. Bienestar animal durante el transporte y su relación con la calidad de la carne bovina. Rev .MVZ Córdoba 17(1): 2936-2944. Disponible en: URL: http://revistas.unicordoba. edu.co/revistamvz/mvz-171/N17N1A18.pdf

SCHWARTZKOPF, K.; HALEY, B.; CHURCH, S.; WOODS J.; O’BYRNE T. 2008. An education and training programmer for livestock transporters in Canada. Veterinaria Italiana 44(1): 273-283. Disponible en: URL: http://www.fao.org/fileadmin/user upload/animalwelfare/An\%20education\%20and\%20training\%20 progr_for\%20livestock\%20transporters\%20in\%20Canada.pdf

TAFUR, M.; ACOSTA, M. Bienestar animal: nuevo reto para la ganadería. ICA Junio, 2006 [consultada en junio del 2016]. Disponible en: URL: http://www.ica.gov.co/getattachment/79b98e64-a258-46d59ce11375a8312434/Publicacion- 20.aspx 